This item was submitted to Loughborough's Research Repository by the author.

Items in Figshare are protected by copyright, with all rights reserved, unless otherwise indicated.

\title{
Diffusion dynamics of defects in Fe and Fe-P systems
}

PLEASE CITE THE PUBLISHED VERSION

PUBLISHER

(C) American Physical Society

LICENCE

CC BY-NC-ND 4.0

\section{REPOSITORY RECORD}

Gordon, Stewart M.J., Steven D. Kenny, and Roger Smith. 2019. "Diffusion Dynamics of Defects in Fe and Fep Systems". figshare. https://hdl.handle.net/2134/1749. 


\title{
Diffusion dynamics of defects in Fe and Fe-P systems
}

\author{
Stewart M. J. Gordon, S. D. Kenny, and Roger Smith \\ Department of Mathematical Sciences, Loughborough University, Loughborough, Leicestershire, LE11 3TU, United Kingdom
}

(Received 14 July 2005; published 2 December 2005)

\begin{abstract}
The dimer method with the Ackland EAM potential has been used to determine the diffusion mechanisms of isolated defects in the bulk of $\alpha$-Fe. Three defect systems were studied, an isolated vacancy, a P-vacancy complex and a $\mathrm{P}$ interstitial defect. Using an event table consisting of the transitions found using the dimer method, the kinetic Monte Carlo method has been used to simulate the diffusion of these defects. Periodic boundary conditions were used to simulate Fe crystals with finite concentrations of $\mathrm{P}$ atoms between 0.006 at. $\%$ and 0.038 at. \%. At lower temperatures of around $350 \mathrm{~K}$, substitutional $\mathrm{P}$ atoms in $\mathrm{Fe}$ act as centers of attraction for vacancy defects, such that the defect moves as a P-vacancy complex for most of the time. However, as the temperature is increased, the phosphorus atom and the vacancy spend greater amounts of time dissociated. We found that $\mathrm{P}$ interstitial defects can also diffuse through the lattice. Diffusion constants have been calculated for these systems at various temperatures and $\mathrm{P}$ concentrations. These showed that an Fe-P dumbbell is the most mobile of these defect systems and a P-vacancy complex the least mobile. For the isolated vacancy and $\mathrm{P}$ interstitial defect systems, the diffusion constant was found to satisfy the Arrhenius relation; the P-vacancy complex, however, showed a deviation from this relation.
\end{abstract}

DOI: 10.1103/PhysRevB.72.214104

\section{INTRODUCTION}

It is well-known that impurity elements are present in various concentrations in all steels and therefore in the steel structures of nuclear pressure vessels (NPVs). When such a vessel is subjected to radiation or enhanced temperature these impurities become mobile. An especially important problem is the segregation of $\mathrm{P}$ atoms to the grain boundaries of the steel, which is thought to be a major cause of embrittlement. ${ }^{1-4}$ This can lead to a serious reduction in the lifetime of NPVs. It is therefore very important to be able to understand the mechanisms by which this segregation takes place, both to predict the mechanical properties of the NPVs as a function of time and to give pointers towards measures that could be taken to reduce the embrittlement problem. To estimate the changes in properties that occur over time at grain boundaries, engineers often use rate theory models. ${ }^{5}$ These can give useful information but require the diffusion rates of the various impurity elements as input parameters. These are often not known experimentally.

To understand better the effect of radiation on the impurity elements in NPVs, molecular dynamics (MD) simulations have been carried out. ${ }^{6}$ These simulations were performed in $\alpha$-Fe with a certain concentration of substitutional $\mathrm{P}$ impurities. The simulations showed that the $\mathrm{P}$ atoms could be displaced from their lattice sites to form Fe-P interstitial dumbbell defects. In addition, interstitial $\mathrm{Fe}$ atoms produced by the collision cascade could be attracted to the $\mathrm{P}$ atoms in the lattice by the large strain field existing around the impurities, leaving vacancies in the region of the cascade core. The interstitial Fe clusters around the substitutional $\mathrm{P}$ atom were found to be very stable, with Fe atoms within the cluster exchanging places but no motion of a $\mathrm{P}$ atom from the substitutional site could be found. The exchange of a substitutional $\mathrm{P}$ atom with an adjacent site in a perfect Fe lattice also involves a very large energy barrier to effect the transition. The $\mathrm{P}$ atoms that were displaced from their substitu-
PACS number(s): 61.82.Bg, 61.72.Ji, 61.72.Cc, 61.80.Az

tional sites to interstitial positions were more mobile and were found to diffuse through the lattice via an intermediate tetrahedral site overcoming an energy barrier of approximately $0.3 \mathrm{eV}$ in the process.

It has also been proposed that substitutional $\mathrm{P}$ atoms could diffuse through the lattice more easily if they were situated close to a vacancy. ${ }^{7}$ If this were to be the case then the production of vacancies by the collision cascades would be an important factor in the enhancement of $\mathrm{P}$ segregation. Thus the mechanisms by which the $\mathrm{P}$ atoms diffuse are not well understood and the problem will be addressed in detail in this paper with the intention of determining the diffusion constants for the most common mechanisms.

Ideally it would be useful to be able to track the defects produced by a collision cascade by using MD. However, cascades are typically terminated after tens of picoseconds because of limitations on computing time, whereas defect motion is an infrequent event on the time scale of a typical MD step. As such, in many cases further investigation cannot be done even using the fastest computer equipment available today. Moreover, even in cases where diffusion events are frequent enough to study using MD, the accessible time scale limits how accurately the diffusivity can be determined.

To address this problem, several accelerated dynamics methods have been developed. ${ }^{8}$ Some of these, such as parallel replica dynamics, ${ }^{9}$ hyperdynamics, ${ }^{10}$ and temperature accelerated dynamics, ${ }^{11}$ work by simulating an MD trajectory in a way that reduces the time taken for transitions to occur. These techniques are based on transition state theory (TST), ${ }^{8,12}$ a model that describes the dynamics of a system in terms of transitions between metastable states. This theory makes it possible to estimate actual transition times from the times that the transitions take under the accelerated conditions. A useful approximation to TST is harmonic transition state theory (hTST), ${ }^{13}$ which provides a simple means of estimating the transition probabilities and escape times if the energy barriers are known. 
Kinetic Monte Carlo (KMC) is a different kind of approach from other accelerated dynamics methods in that there is no MD trajectory where the system is evolved in real time. Instead the simulation is event driven. At each step, a transition is randomly selected from those that are possible from the current state. TST or hTST is then used to determine the relative probabilities of the transitions and the typical time taken for them to occur. The system is then advanced to the new state, and the simulation time clock incremented by the appropriate amount.

There are two main types of KMC simulation. The first kind $^{14}$ uses an event table generated in advance, by considering all possible system states and identifying transitions between them. Often when this is done, only states in which all atoms are in lattice sites are considered, limiting the realism of the predicted evolution of the system. Moreover, in systems with many states, it can be difficult or even impossible to build an adequate event table for KMC. ${ }^{8}$

The alternative is on-the-fly KMC (OFKMC) ${ }^{18}$ in which the event table is built up as the simulation progresses. From each state that the system visits, a set of possible transitions is generated and used to advance the system to a new state. A typical technique for discovering the transitions from each state is the dimer method. ${ }^{15}$ This algorithm makes no assumptions about the types of transition mechanisms that will be found in the system, and so can be used to find transitions that would be overlooked if searching using molecular statics ${ }^{16}$ or the nudged elastic band method. ${ }^{17}$ Since only the states actually visited are considered, OFKMC is much more feasible than using a predefined event table in systems of many states. Examples of such systems to which OFKMC has been applied include island ripening and crystal growth. ${ }^{18,19}$ However, it is computationally expensive for large systems, and has so far only been applied to surfaces, where the number of free atoms required to model the transitions is much less than for bulk problems.

This paper presents the result of using KMC with a predefined event table, built up by using the dimer method, to model diffusion of three kinds of defects commonly found in irradiated $\alpha$-Fe crystals: ${ }^{6}$ an isolated vacancy, a complex of a vacancy and a substitutional $\mathrm{P}$ atom, and an interstitial defect created by adding a $\mathrm{P}$ atom. Since there is a relatively small number of distinct states in each case, the use of a predefined event table is suited to these simple systems.

A commonly used measure of the rate at which a defect diffuses is the diffusion constant. For a typical diffusion system, the diffusion constant varies with temperature according to the Arrhenius relation

$$
D=D_{0} e^{-E / k_{B} T},
$$

where $D_{0}$ and $E$ are system-dependent parameters. One of the aims of this work is to determine whether this relation holds for the systems being studied and to calculate the values of $D_{0}$ and $E$.

\section{METHODOLOGY}

In order to apply hTST to determine the transition probabilities and mean escape times, it is necessary to calculate the energy barriers for the transitions. We have used the dimer method ${ }^{15}$ to find the transition points in the system and their associated energy barriers. Algorithms such as the nudged elastic band method ${ }^{17}$ can be used to calculate transition barriers between known initial and final states. The dimer method differs in that it requires only the initial state; hence its importance lies in its being able to find transitions that were not presupposed to exist, including cooperative transitions, which are transitions that rely on two or more atoms changing position simultaneously. It also differs from earlier mode-following algorithms $\mathrm{s}^{20,21}$ in that it uses only the first derivative of the potential function, hence avoiding the difficulties and computational cost of evaluating the Hessian matrix. $^{15}$

The dimer method is a numerical algorithm that works by finding saddle points in a potential energy surface of arbitrarily high dimension. The potential surface of interest is the $3 \mathrm{~N}$-dimensional surface of all possible configurations of the $N$ atoms in the system. Basins of this surface correspond to metastable states of the system, and the saddle points are the lowest-energy transition points between states. By locating these saddle points and calculating their energy levels, we can therefore determine the minimum energy barrier for a transition to take place.

The dimer method works by manipulating two points on the potential surface (the dimer), a small constant distance apart in a variable direction (the dimer vector). Starting near a local minimum of the surface, the algorithm adjusts the dimer vector so that the dimer lies along a line of lowest curvature of the potential surface, and then translates along this line. By repeating these steps, the dimer converges towards the saddle point. The transitions were then traced to their initial and final states by using a conjugate gradient algorithm to minimize the potential energy of the system, with starting points either side of the transition point.

We used system sizes of $14 \times 14 \times 14$ bcc unit cells (5488 lattice points) in the dimer searches, generally with 200 to 500 atoms free to move. On the remaining atoms in the lattice, the forces, and hence the dimer vector components, are treated as zero. On each initial state we generally carried out 100 dimer searches with different initial dimer vectors. These initial vectors were generated randomly to have nonzero components only on a 40- to 100 -atom subset of the free atoms. Performing many searches made it possible both to find a variety of transitions and to compare energy barrier approximations on searches from different initial vectors leading to the same transition, thus getting an idea of how accurate the energy barriers are. When using the dimer method on bulk material, we found that more free atoms are needed for accurate energy barriers than for surface problems previously studied. ${ }^{15}$ Thus we adapted the dimer method to periodically relax the fixed atoms using the conjugate gradient method. This was found to yield higher success rates than simply increasing the number of free atoms, and to give energy barriers converged to the $10^{-5} \mathrm{eV}$ level.

The interactions between atoms were modeled using the Ackland EAM potential for $\alpha$-Fe containing P.22 This is a many-body potential designed to take into account defect structures commonly found in collision cascades. It is a single potential that considers $\mathrm{Fe}$ and $\mathrm{P}$ atoms together, in 


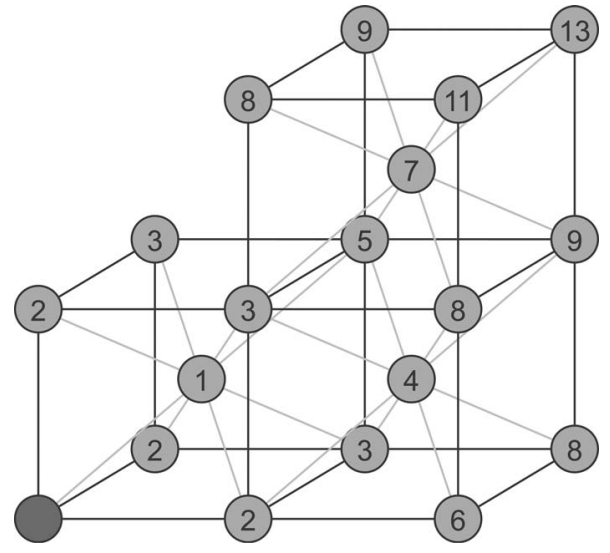

FIG. 1. Neighbor numbers corresponding to small displacements between lattice points.

contrast to the older Ackland $\mathrm{Fe}^{23}$ and Morse Fe-P and P-P pairwise potentials ${ }^{24}$ used in previous studies. ${ }^{25}$

The transition times are calculated from the energy barriers using hTST. This is an approximate model based on TST which requires that the energy barriers are known. In this approach, we calculate the rate constant for each transition, based on the transition's energy barrier and the temperature of the system. The rate constant of a transition is the mean frequency of the transition over the time that the system spends in the transition's initial state. For a transition from state $i$ to state $j$, the rate constant is given by the Arrhenius equation

$$
r_{i j}=\nu e^{-E_{i j} / k_{B} T}
$$

where $E_{i j}$ is the transition energy barrier, $k_{B}$ is Boltzmann's constant, $T$ is the temperature and $\nu$ is the attempt frequency. For this work, we have used the value $\nu=10^{13} \mathrm{~s}^{-1}$, which is a typical value assumed for rate theory modelling. ${ }^{26}$ The rates at which transitions occur, and hence the diffusion constants, are linearly dependent upon this value.

In the hTST model, the time for which the system remains in a given state is exponentially distributed and independent of the state to which the system is to exit. The mean of this distribution, for a state $i$, is

$$
\tau_{i}=\frac{1}{\sum_{j} r_{i j}},
$$

where the sum is taken over all possible transitions from state $i$. Consequently, given a second state $j$, the probability that a given transition is next to occur is proportional to its rate constant and is given by

$$
p_{i j}=r_{i j} \tau_{i} .
$$

To increment the simulation clock, we then use the formula

$$
\Delta t=-\tau_{i} \ln R,
$$

where $R$ is a random variable with uniform distribution over the interval $(0,1]$.

Using an event table consisting of the states and transitions that we discovered in the system, we ran KMC simu-
TABLE I. Diffusion constants for an isolated vacancy in Fe.

\begin{tabular}{cc}
\hline \hline Temperature $(\mathrm{K})$ & Diffusion constant $\left(\mathrm{m}^{2} \mathrm{~s}^{-1}\right)$ \\
\hline 350 & $2.5 \times 10^{-16}$ \\
400 & $3.6 \times 10^{-15}$ \\
500 & $1.5 \times 10^{-13}$ \\
600 & $1.8 \times 10^{-12}$ \\
700 & $1.0 \times 10^{-11}$ \\
\hline \hline
\end{tabular}

lations at various temperatures. Diffusion constants were calculated by using the standard equation

$$
D=\frac{\left\langle r^{2}\right\rangle}{6 t},
$$

where $r$ is the distance of the defect from its initial point over a sample time interval $t$. It is necessary to take many sample intervals to obtain an accurate value of the diffusion constant. For the isolated vacancy system, the location of the vacancy was used to calculate the diffusion constant. For the other systems, two diffusion constants were calculated, that of the P atom and that of the vacancy or interstitial defect. At each temperature, at least 10000 samples were taken, in which the total number of events observed is generally of the order of $10^{10}$. This was found to give the diffusion constant converged to two significant figures. The frequency of events, and hence the total simulation time, was varied with temperature. At $350 \mathrm{~K}$, where events are infrequent, we found that samples were best taken at millisecond intervals, giving a simulation time of $10 \mathrm{~s}$. At higher temperatures, events happen more frequently, so shorter sampling intervals

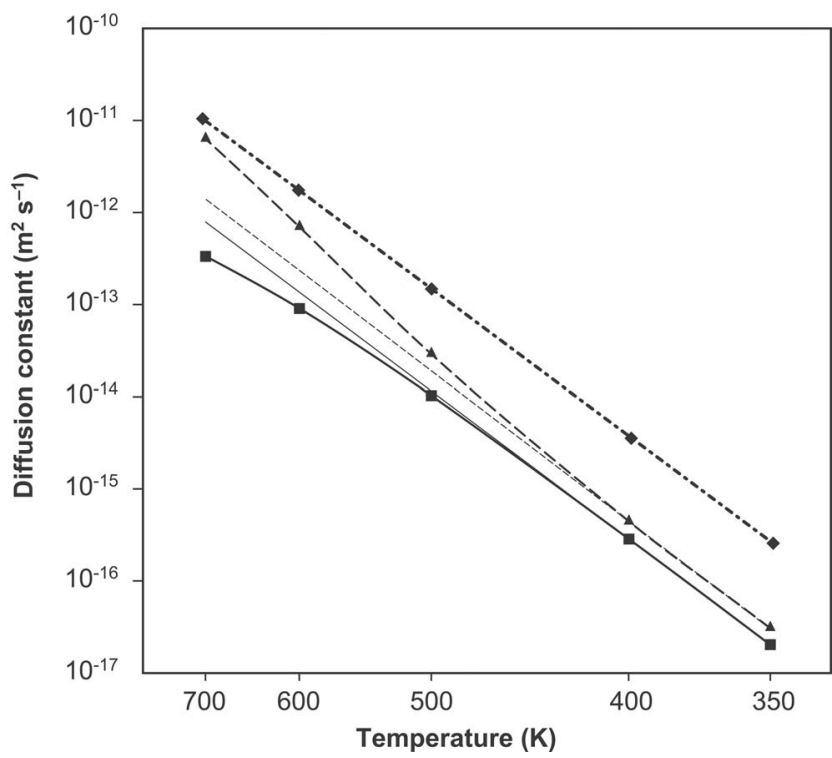

FIG. 2. Arrhenius plot of the diffusion constants of an isolated vacancy in Fe ( $\bullet$, dashed-dotted line), and of a P atom ( $\boldsymbol{\square}$, solid line) and a vacancy ( $\boldsymbol{\Lambda}$, dashed line) in a P-vacancy complex with a periodic cell size of $15 \times 15 \times 15$ unit cells. The fine lines show the extrapolation from the diffusion constants in the P-vacancy system calculated at $350 \mathrm{~K}$ and $400 \mathrm{~K}$ if the Arrhenius relation is assumed. 
(a) First neighbor P-vacancy

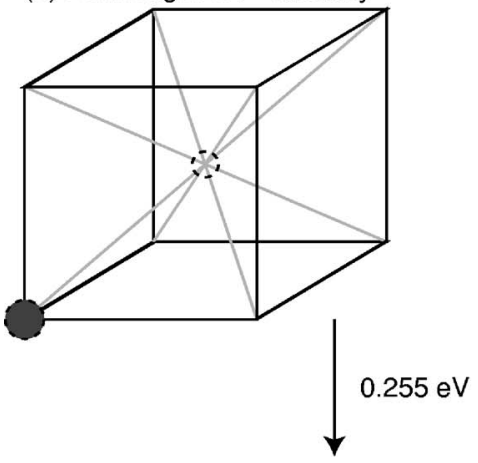

(b)

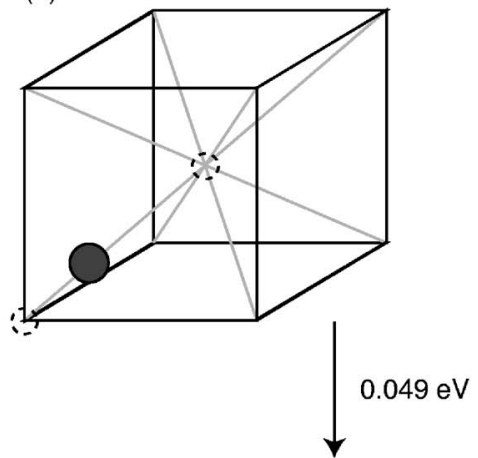

(c)

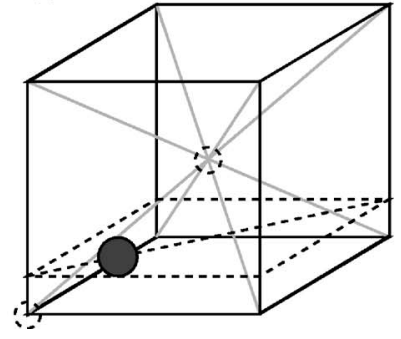

(f) First neighbor vacancy-P

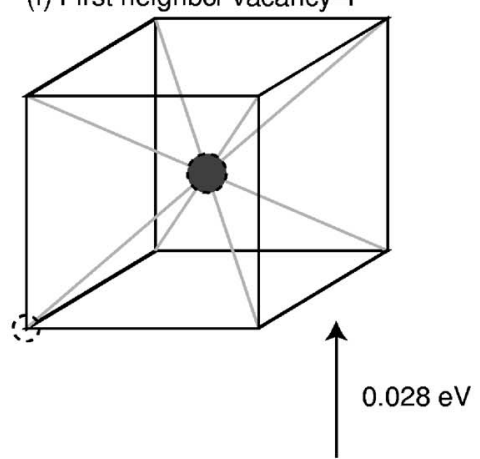

(e)

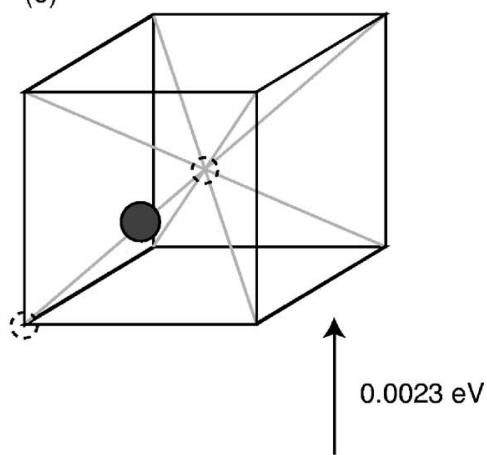

(d)

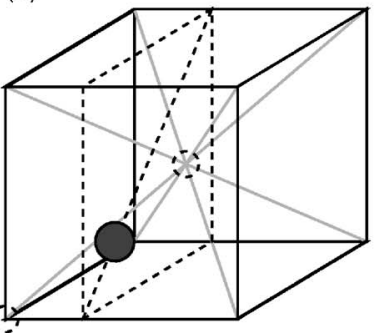

FIG. 3. The mechanism of the first neighbor P-vacancy exchange in $\mathrm{Fe}$, with energy barriers. A single bcc unit cell, which for $\alpha$-Fe has side length $2.86 \AA$, is shown. From the first neighbor P-vacancy state (a), the $\mathrm{P}$ atom moves about $0.9 \AA$ towards the vacancy (b). It then moves to a point with coordinates $(0.7,0.7,0.4) \AA$ relative to its initial lattice point $(\mathrm{c})$. The sequence of states is then reversed (d), (e), (f). (down to $10 \mu \mathrm{s}$ ) and total simulation times of about $0.1 \mathrm{~s}$ were found to be adequate.

When running the KMC simulations for the P-vacancy complex and $\mathrm{P}$ interstitial systems, it was necessary to consider that the vacancy or interstitial defect may become dissociated from the $\mathrm{P}$ atom. The defect then behaves as an isolated vacancy or self-interstitial defect in Fe, i.e., it is not influenced by the $\mathrm{P}$ atom. This possibility also means that the defect can become dissociated and then never return to the vicinity of the $\mathrm{P}$ atom. This was addressed by using periodic boundary conditions (PBC) to represent a bulk crystal with a finite concentration of $\mathrm{P}$ atoms. A periodic cell size of 11 $\times 11 \times 11$ bcc unit cells gives a $\mathrm{P}$ concentration of 0.038 at. \%, which is a typical concentration found in NPV steels. Using periodic cell sizes between $11 \times 11 \times 11$ and $20 \times 20 \times 20$, we studied $\mathrm{P}$ concentrations down to 0.006 at. $\%$.

In enumerating the transitions, it is necessary to consider the possible positions of the vacancy or the interstitial defect relative to the $\mathrm{P}$ atom. These positions are identified by neighbor numbers, determined by the ranking order of the distance between the lattice point of the vacancy or interstitial defect and that of the $\mathrm{P}$ atom. The first neighbors are thus adjacent atoms along $\langle 111\rangle$ directions, which are separated by a distance of $2.48 \AA$. The next nearest atoms are the sec- ond neighbors, with a separation distance equal to the bcc unit cell size of $2.87 \AA$. The neighbor numbers corresponding to various lattice displacements are given in Fig. 1.

The PBC approximation is limited in that it treats the distribution of $\mathrm{P}$ atoms as being regular and does not distinguish the positions of different $\mathrm{P}$ atoms. A more accurate approach would be to consider a cell containing several, randomly distributed $\mathrm{P}$ atoms; however, this technique is more complex as it becomes necessary to consider that two $\mathrm{P}$ atoms may become sufficiently close to each other that they either interact with each other or simultaneously interact with the vacancy or interstitial.

\section{RESULTS}

\section{A. Isolated vacancy}

In the isolated vacancy system, the defect was found to alternate between two states, the vacancy state itself and a metastable split vacancy state with an Fe atom positioned midway between two vacant lattice sites. From the simple vacancy state, one of the eight Fe atoms nearest the vacancy moves into the split vacancy position, with an energy barrier of $0.640 \mathrm{eV}$. The split vacancy then transitions back into a simple vacancy with an energy barrier of $0.091 \mathrm{eV}$, by the $\mathrm{Fe}$ 


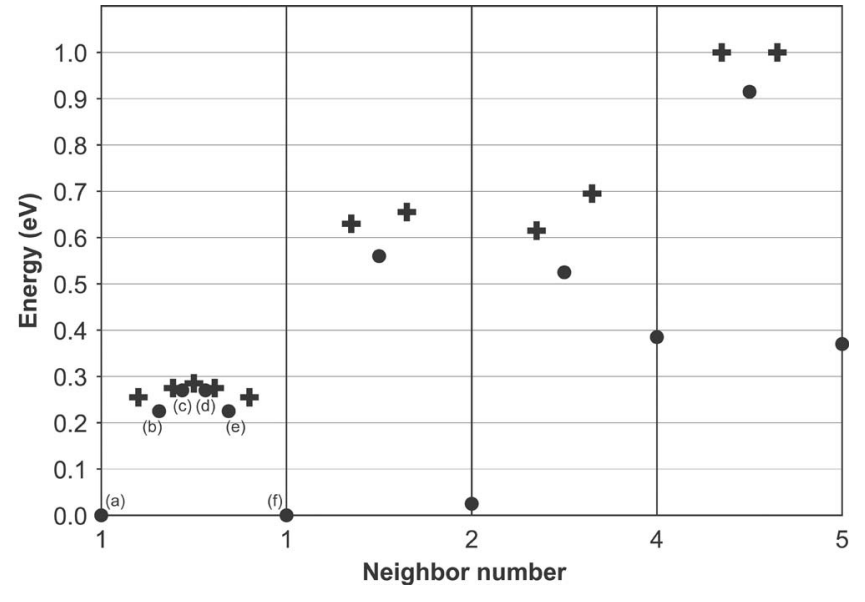

FIG. 4. The energy profiles of the P-vacancy exchange mechanism and three Fe-vacancy exchange mechanisms between different neighbor sites of the $\mathrm{P}$ atom. Only the metastable states $(\boldsymbol{)})$ and the saddle points $(+)$ are plotted. The letters on the profile of the P-vacancy exchange refer to the stages of the exchange illustrated in Fig. 3.

atom moving either back to its previous position or into the vacancy of the previous state, leaving its own previous lattice site vacant.

By running the KMC simulation on the isolated vacancy system, diffusion constants were calculated (Table I). Figure 2 shows that this system obeys the Arrhenius relation; we calculated the values $D_{0}=4.2 \times 10^{-7} \mathrm{~m}^{2} \mathrm{~s}^{-1}$ and $E=0.64 \mathrm{eV}$.

\section{B. P-vacancy complex}

In the P-vacancy complex, transitions were found out to states in which the $\mathrm{P}$ atom and vacancy are separated at up to thirteenth neighbor positions (a distance of 8.6 $\AA$ ). Beyond the eighth neighbor position, the energy barrier for vacancy diffusion had converged to within $0.01 \mathrm{eV}$ of that for diffusion of an isolated vacancy, leading to only very small differences in the transition probabilities and times. The vacancy was therefore considered to have dissociated when it passed beyond the eighth neighbor position (a distance of $6.8 \AA$ A).

The mechanism most frequently observed is the exchange of the $\mathrm{P}$ atom and vacancy when they are at first neighbor positions, via a sequence of metastable states (Fig. 3). From the starting position (a), the $\mathrm{P}$ atom moves in the $\langle 111\rangle$ direction towards the vacancy (b) and then moves to a position slightly off the line connecting the two lattice points (c). To complete the exchange, the system passes through same three states in reverse order (d), (e), (f) with the P atom now in the area of the original vacancy. Figure 4 shows the energy profile of this mechanism. Overall, the system overcomes an energy barrier of $0.287 \mathrm{eV}$ during this exchange. At a temperature of $300 \mathrm{~K}$, only the transitions corresponding to this mechanism were observed, hence the defect did not diffuse through the lattice.

Other mechanisms enable the P-vacancy complex to reorient itself by Fe-vacancy exchanges around the $\mathrm{P}$ atom. The most common of these reorientation mechanisms is via a state in which the vacancy is a second neighbor of the $\mathrm{P}$ atom (Fig. 5). From the first neighbor position (a), an $\mathrm{Fe}$ atom from a second neighbor position moves to form a split vacancy configuration (b). From this metastable position, the $\mathrm{Fe}$ atom moves into the first neighbor position (c), leaving a vacancy at the second neighbor position. From this position, an Fe atom from a first neighbor position in an adjacent cell moves towards the second neighbor position (d), (e). A split vacancy state was found to be metastable in every transition mechanism between P-vacancy positions except for that between the first and third neighbor positions. The energy barriers of transitions between different neighbor positions are listed in Table II. Figure 4 also illustrates the energy profiles of three of these Fe-vacancy exchange mechanisms.

The P-simple vacancy states all have energy levels less than $0.5 \mathrm{eV}$ above that of the first neighbor P-vacancy state. Some of these energy levels are given in Table III. It should therefore be expected that the system explores a wide variety of states. Some agreement has been seen in the energy levels and barriers with those calculated by Ackland ${ }^{22}$ and with $a b$ initio results. ${ }^{27}$

We ran KMC simulations on two different periodic cell sizes at five different temperatures, and calculated the diffusion constants. The first of these is a cell of $11 \times 11 \times 11$ unit cells $(31.5 \AA)$, representing a P concentration of 0.038 at. \% . The results are shown in Table IV. The $\mathrm{P}$ atom diffusion constants calculated at this cell size for $500 \mathrm{~K}$ and $600 \mathrm{~K}$ agree with those calculated by Barashev. ${ }^{28}$ The second is a cell of $15 \times 15 \times 15$ unit cells $(43.0 \AA)$, representing a P concentration of 0.015 at. \%; the results at this concentration are given in Table V. As shown in Fig. 2, the P-vacancy system was found not to satisfy the Arrhenius relation.

We also compared the relative amounts of time for which the vacancy is associated and dissociated with the $\mathrm{P}$ atom (Tables IV and V). The amount of time over a given interval for which the system is dissociated increases with temperature. This shows that, while the vacancy is strongly attracted to a substitutional $\mathrm{P}$ atom, the effect of this attraction weakens as the temperature increases. This is as expected, since the higher-energy transitions are more probable at higher temperatures.

This increase in the time that the $\mathrm{P}$ atom and vacancy spend dissociated also means that, as the temperature increases, there is less time in which the $\mathrm{P}$ can diffuse. On the other hand, the vacancy is more mobile when dissociated than when moving in the P-vacancy complex. This accounts for the non-Arrhenius behavior, since as dissociation time increases, the overall P mobility is decreased, and the overall vacancy mobility is increased, compared with what would otherwise be expected with the variation in temperature.

At a temperature of $500 \mathrm{~K}$, we compared the diffusion constants at five different periodic cell sizes, representing $\mathrm{P}$ concentrations from 0.038 at. $\%$ down to 0.006 at. $\%$. The results are in Table VI. As the $\mathrm{P}$ concentration decreases, the diffusion constant of the $\mathrm{P}$ atom decreases, and the diffusion constant of the vacancy increases. The time for which the vacancy and $\mathrm{P}$ atom are associated also decreases at lower $\mathrm{P}$ concentrations. This illustrates that when the $\mathrm{P}$ concentration is lower, the vacancy diffuses further before associating with another $\mathrm{P}$ atom. It follows that the vacancy is more mobile, 


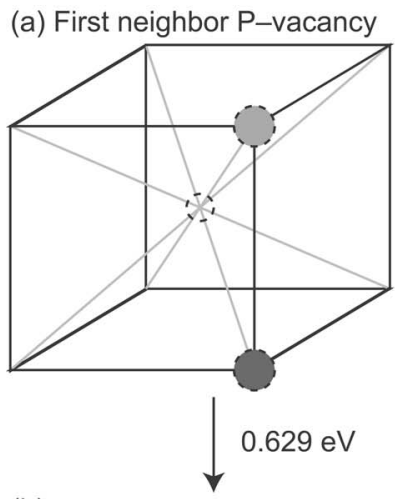

(b)
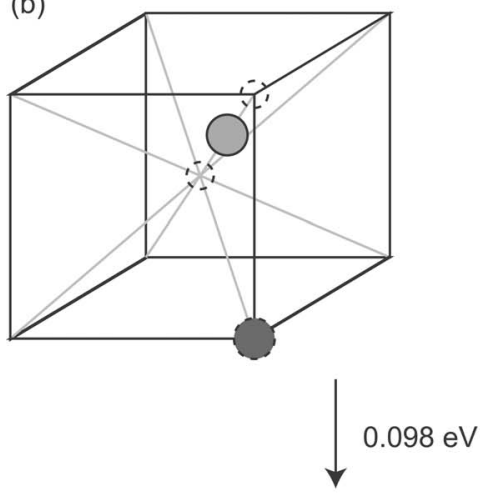

(e) First neighbor P-vacancy

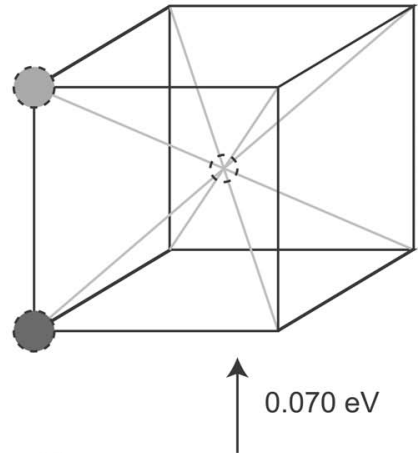

(d)

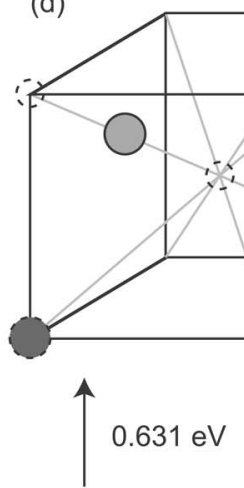

(c) Second neighbor P-vacancy

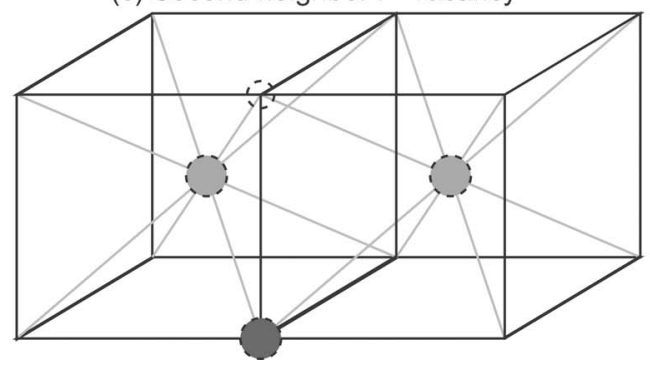

hence the increasing diffusion constants, but the $\mathrm{P}$ atom has less opportunity to diffuse, leading to a decreased diffusion constant.

\section{P interstitial defect}

In the $\mathrm{P}$ interstitial defect system, the lowest-energy configuration is a $\langle 110\rangle \mathrm{Fe}-\mathrm{P}$ mixed dumbbell. In the most common diffusion mechanism, the $\mathrm{P}$ atom moves to either of two tetrahedral sites with an energy barrier of $0.263 \mathrm{eV}$. From this position, it can move to any of four $\langle 110\rangle$ Fe-P dumbbell states, including the one from which it came, with a barrier of $0.050 \mathrm{eV}$. A sequence of moves of this type is given in Fig. 6.

The $\langle 110\rangle$ Fe-P dumbbell can also transition into a $\langle 110\rangle$ $\mathrm{Fe}-\mathrm{Fe}$ dumbbell about a site that is first neighbor to the $\mathrm{P}$ atom, with a barrier of $0.597 \mathrm{eV}$. From this state, the Fe-Fe dumbbell configuration can migrate to other lattice sites around the $\mathrm{P}$ atom. Transitions have been found out to states in which the $\mathrm{P}$ atom and dumbbell are up to tenth neighbor positions. Most transitions found in the system were between $\langle 110\rangle \mathrm{Fe}-\mathrm{Fe}$ dumbbell states at adjacent lattice sites, with the initial and final dumbbells in perpendicular (100) planes

TABLE II. Transition barriers found in the P-vacancy system. The state numbers refer to neighbor numbers given in Fig. 1. The transition between the first and third neighbor states was not found to have an intermediate state. Hence for this case only one energy barrier is given in each direction.

\begin{tabular}{ccccc}
\hline \hline & & \multicolumn{2}{c}{ Energy barriers $(\mathrm{eV})$} \\
\cline { 3 - 4 } Initial state & Final state & \multicolumn{2}{c}{ Initial $\rightarrow$ intermediate } & Intermediate $\rightarrow$ final \\
\hline 1st & 2nd & 0.629 & 0.098 \\
1 st & 3rd & & 1.065 & \\
1st & 5th & 0.582 & & 0.388 \\
2nd & 1st & 0.631 & & 0.070 \\
2nd & 4th & 0.592 & 0.165 \\
3rd & 1st & & 0.632 & \\
3rd & 4th & 0.622 & & 0.076 \\
4th & 2nd & 0.307 & 0.090 \\
4th & 3rd & 0.646 & 0.099 \\
4th & 5th & 0.615 & 0.093 \\
5th & 1st & 0.528 & 0.071 \\
5th & 4th & 0.637 & 0.086 \\
\hline \hline
\end{tabular}

FIG. 5. The mechanism of the first neighbor P-vacancy reorientation via the second neighbor state, with energy barriers. From the first neighbor P-vacancy state (a), an Fe atom (light sphere) at a second neighbor position moves to form a split vacancy (b) before filling the first neighbor site, leaving the vacancy and $\mathrm{P}$ atom (dark sphere) in second neighbor positions. The system can then return to the first neighbor P-vacancy state by a similar sequence of moves (d), (e). 
TABLE III. Potential energy levels of states with the vacancy separated at different distances from the $\mathrm{P}$ atom in the P-vacancy system. The first neighbor state has been taken as a base level.

\begin{tabular}{cc}
\hline \hline State & Energy level $(\mathrm{eV})$ \\
\hline 1st & 0.000 \\
2nd & 0.026 \\
3rd & 0.433 \\
4th & 0.385 \\
5 th & 0.371 \\
6th & 0.360 \\
7th & 0.368 \\
\hline \hline
\end{tabular}

(Fig. 7). Unlike the P-Fe dumbbell migration and most vacancy moves, generally no intermediate state was seen between successive Fe-Fe dumbbell configurations. Some energy barriers of these transitions are given in Table VII. In this table and the following descriptions of dumbbells, the first direction refers to the orientation of the dumbbell, and the second one gives the direction of the displacement of the dumbbell center from the $\mathrm{P}$ atom. All of these transitions, except for those between [110] at first neighbor [111] and [011] at second neighbor [100], and between [110] at first neighbor [111] and [110] at third neighbor [110], are of the form of Fig. 7.

Unlike in the P-vacancy system, there are relatively few states with low energy levels relative to the $\langle 110\rangle$ Fe-P dumbbell state (Table VIII). Most Fe-Fe dumbbell states have energy levels at least $1 \mathrm{eV}$ above that of the Fe-P dumbbell. This implies that an interstitial defect is more strongly attracted to a P atom than a vacancy is. It should therefore be expected that the system spends most of its time moving between the Fe-P dumbbell and tetrahedral states. Also, since the main energy barriers for P migration are lower than those involved in the P-vacancy system, and it also takes fewer events for the $\mathrm{P}$ atom to move the same distance, it is to be expected that the $\mathrm{P}$ atom will diffuse more rapidly by moving as an interstitial defect than as a P-vacancy complex.

When the interstitial defect is dissociated from the $\mathrm{P}$ atom, the $\langle 110\rangle$ dumbbell migration shown in Fig. 7 is the most common transition, with a barrier of $0.304 \mathrm{eV}$, which is similar to the barrier calculated $a b$ initio and experimentally. ${ }^{29} \mathrm{~A}$ transition has also been found from the $\langle 110\rangle$ dumbbell state to an octahedral state, with a barrier of $0.676 \mathrm{eV}$; the reverse transition has barrier $0.034 \mathrm{eV}$.

At all temperatures, the KMC simulations showed that the interstitial defect spends over $98 \%$ of its time in the $\langle 110\rangle$ Fe-P dumbbell state, with the $\mathrm{P}$ atom migrating via the $\mathrm{P}$ tetrahedral state. At $350 \mathrm{~K}$ and $400 \mathrm{~K}$, the system was moving between these two states for over $99.99999 \%$ of the time, becoming an $\mathrm{Fe}-\mathrm{Fe}$ dumbbell with the $\mathrm{P}$ atom in a substitutional position for only a few transition events at a time. Consequently the interstitial defect did not become dissociated from the $\mathrm{P}$ atom.

This system has previously been studied using preliminary MD simulations. ${ }^{22}$ At $1200 \mathrm{~K}$, the interstitial defect dissociated from the $\mathrm{P}$ atom after $80 \mathrm{ps}$; however, at $600 \mathrm{~K}$ no
TABLE IV. Diffusion constants and association times for the P-vacancy complex with a PBC cell of $11 \times 11 \times 11$ bcc unit cells, representing a 0.038 at. $\% \mathrm{P}$ concentration. The time associated is defined here as the percentage of the time for which the vacancy was within $6.8 \AA$ of the $\mathrm{P}$ atom, i.e., at up to the eighth neighbor position.

\begin{tabular}{cccc}
\hline \hline & \multicolumn{2}{c}{ Diffusion constants $\left(\mathrm{m}^{2} \mathrm{~s}^{-1}\right)$} & \\
\cline { 2 - 3 } Temperature $(\mathrm{K})$ & P atom & Vacancy & Time associated \\
\hline 350 & $2.3 \times 10^{-17}$ & $1.2 \times 10^{-16}$ & $99.8 \%$ \\
400 & $3.2 \times 10^{-16}$ & $1.3 \times 10^{-15}$ & $99.4 \%$ \\
500 & $1.1 \times 10^{-14}$ & $1.9 \times 10^{-14}$ & $95.2 \%$ \\
600 & $1.1 \times 10^{-13}$ & $4.1 \times 10^{-13}$ & $83.9 \%$ \\
700 & $5.1 \times 10^{-12}$ & $4.1 \times 10^{-12}$ & $66.9 \%$ \\
\hline \hline
\end{tabular}

dissociation was seen. However, by extending the simulation beyond the accessible MD time scale, we have seen that dissociation can occur at temperatures as low as $500 \mathrm{~K}$, at which the interstitial went beyond the sixth neighbor position approximately 60 times per second, but only for short periods of the order of nanoseconds. At higher temperatures dissociations are more frequent, but even at $700 \mathrm{~K}$, the dissociation was for only $0.006 \%$ of the time.

The diffusion constants for this system at a periodic cell size of $11 \times 11 \times 11$ are given in Table IX. In this system, there was no noticeable difference between the diffusion constant of the $\mathrm{P}$ atom and that of the interstitial. There was also no noticeable deviation from the Arrhenius relation, with $D_{0}=1.7 \times 10^{-8} \mathrm{~m}^{2} \mathrm{~s}^{-1}$ and $E=0.26 \mathrm{eV}$.

\section{CONCLUSIONS}

This work has identified diffusion mechanisms in isolated vacancy, P-vacancy and $\mathrm{P}$ interstitial defects in $\alpha$-Fe, and calculated for the first time diffusion constants associated with the most common defect diffusion mechanisms. Comparisons with previous studies using an older interatomic potential ${ }^{25}$ indicate a similar mechanism and barrier for the

TABLE V. Diffusion constants and association times for the P-vacancy complex with a PBC cell of $15 \times 15 \times 15$ bcc unit cells, representing a 0.015 at. $\% \mathrm{P}$ concentration. The time associated is defined here as the percentage of the time for which the vacancy was within $6.8 \AA$ of the $\mathrm{P}$ atom, i.e., at up to the eighth neighbor position.

\begin{tabular}{cccc}
\hline \hline & \multicolumn{2}{c}{ Diffusion constants $\left(\mathrm{m}^{2} \mathrm{~s}^{-1}\right)$} & \\
\cline { 2 - 3 } Temperature $(\mathrm{K})$ & $\mathrm{P}$ atom & Vacancy & Time associated \\
\hline 350 & $2.0 \times 10^{-17}$ & $3.0 \times 10^{-17}$ & $99.4 \%$ \\
400 & $2.8 \times 10^{-16}$ & $4.3 \times 10^{-16}$ & $98.7 \%$ \\
500 & $1.0 \times 10^{-14}$ & $2.8 \times 10^{-14}$ & $88.5 \%$ \\
600 & $8.9 \times 10^{-14}$ & $6.8 \times 10^{-13}$ & $66.9 \%$ \\
700 & $3.3 \times 10^{-13}$ & $6.2 \times 10^{-12}$ & $43.6 \%$ \\
\hline \hline
\end{tabular}


TABLE VI. Diffusion constants for the P-vacancy complex at $500 \mathrm{~K}$ with different periodic cell sizes, representing different concentrations of $\mathrm{P}$ atoms in $\mathrm{Fe}$.

\begin{tabular}{ccccc}
\hline \hline & & \multicolumn{2}{c}{ Diffusion constants $\left(\mathrm{m}^{2} \mathrm{~s}^{-1}\right)$} & \\
\cline { 3 - 4 } $\begin{array}{c}\text { Periodic cell size } \\
\text { (bcc unit cells) }\end{array}$ & P atom concentration & P atom & Vacancy & Time associated \\
\hline 11 & 0.038 at. \% & $1.1 \times 10^{-14}$ & $1.9 \times 10^{-14}$ & $95.3 \%$ \\
13 & 0.023 at. $\%$ & $1.1 \times 10^{-14}$ & $2.2 \times 10^{-14}$ & $92.3 \%$ \\
15 & 0.015 at. $\%$ & $1.0 \times 10^{-14}$ & $2.8 \times 10^{-14}$ & $88.5 \%$ \\
17 & 0.010 at. $\%$ & $9.6 \times 10^{-15}$ & $3.4 \times 10^{-14}$ & $84.1 \%$ \\
20 & 0.006 at. $\%$ & $8.7 \times 10^{-15}$ & $4.5 \times 10^{-14}$ & $76.1 \%$ \\
\hline \hline
\end{tabular}

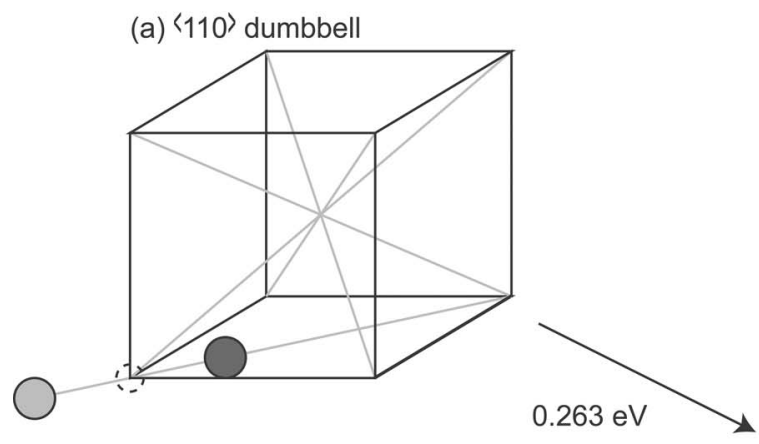

(b) Tetrahedral

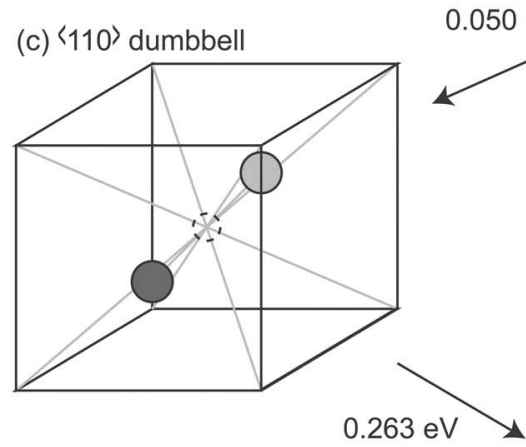

(d) Tetrahedral on plane between cells
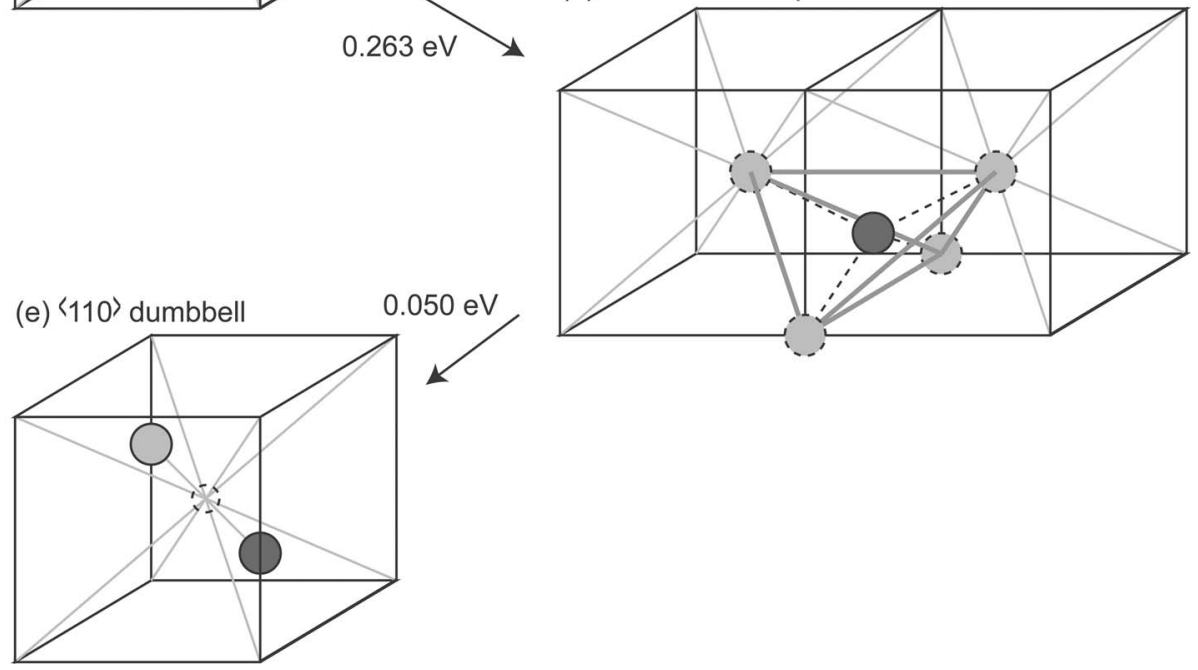

FIG. 6. Migration of the Fe-P $\langle 110\rangle$ dumbbell via a tetrahedral state, with energy barriers. 

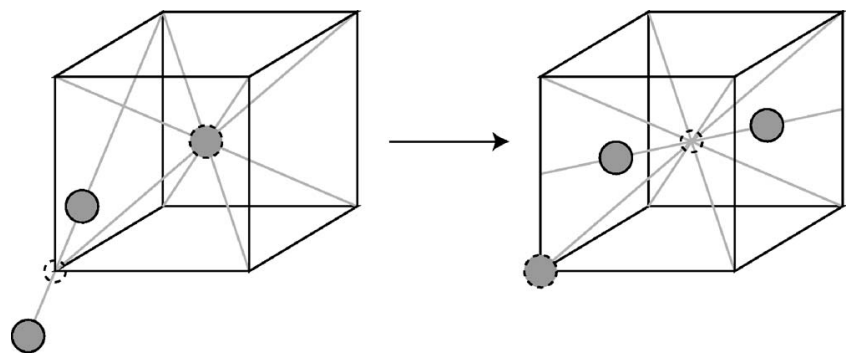

FIG. 7. The most common transition mechanism of an Fe-Fe $\langle 110\rangle$ dumbbell, both in isolation and near a substitutional $\mathrm{P}$ atom. The dumbbell moves to an adjacent lattice site and rotates to a $\langle 110\rangle$ direction in a perpendicular (100) plane to the one in which it started.

$\langle 110\rangle$ Fe-P dumbbell to P tetrahedral transition, but this work has also shown other transitions and barriers in the system. We believe that the interatomic potential used in this work, which has been fitted to more ab initio data, is more sophisticated for the study of P defects in Fe.

We have used KMC to model the diffusion of these defect types. This has made it possible to simulate defect diffusion up to the order of seconds, which would have taken many thousands of years to simulate using classical MD. By doing this, we have calculated the diffusion constants of these defect systems at various temperatures and various $\mathrm{P}$ atom concentrations, to a greater degree of accuracy than is possible under the time constraints of MD. We have thus determined that the diffusion constants of an isolated vacancy and of a P-interstitial defect conform to the Arrhenius relation, but the diffusion of the P-vacancy complex does not obey this law.

In the P-vacancy system we saw that the vacancy is attracted to the $\mathrm{P}$ atom. The effect of this attraction becomes weaker as the temperature increases. This is because the transitions with higher energy barriers become more probable at higher temperatures. The probability distribution of the transitions therefore becomes more balanced, so that from a
TABLE VIII. Potential energy levels of different states in the interstitial defect system, relative to the $\langle 110\rangle \mathrm{Fe}-\mathrm{P}$ dumbbell state. All states except for the first two are $\mathrm{Fe}-\mathrm{Fe}$ dumbbell positions near a substitutional $\mathrm{P}$ atom. For each of these, two direction vectors are given, the orientation of the dumbbell and the direction of the displacement of the dumbbell center from the $\mathrm{P}$ atom.

\begin{tabular}{cc}
\hline \hline State & Energy level $(\mathrm{eV})$ \\
\hline$\langle 110\rangle$ Fe-P & 0.000 \\
Tetrahedral & 0.212 \\
{$[110]$ at 1 st $[111]$} & 0.581 \\
{$[1 \overline{1} 0]$ at 1 st $[111]$} & 1.330 \\
{$[110]$ at 2nd $[100]$} & 1.040 \\
{$[011]$ at 2nd $[100]$} & 1.248 \\
{$[110]$ at 3rd $[110]$} & 1.441 \\
{$[1 \overline{1} 0]$ at 3rd $[110]$} & 1.058 \\
{$[101]$ at 3rd $[110]$} & 1.006 \\
\hline \hline
\end{tabular}

given state the vacancy is more likely to move away from the $\mathrm{P}$ atom and less likely to move towards it than at the lower temperatures. In the $\mathrm{P}$ interstitial defect system, we found higher diffusion constants than those found for the isolated vacancy and P-vacancy complex. This shows that, as expected from the energy levels and barriers involved, the $\mathrm{P}$ interstitial defect is the most mobile of the three defect systems at the temperatures studied, and the P-vacancy complex is the least mobile.

Although the substitutional site is the preferred location of $\mathrm{P}$ in $\mathrm{Fe}$, radiation can dislodge the $\mathrm{P}$ atoms into the interstitial position which is well-separated from vacancies created by the cascade. This implies that the interstitial diffusion mechanism could be an important process by which $\mathrm{P}$ atoms can migrate to grain boundaries in NPVs subjected to radiation damage.

TABLE VII. Transition barriers found between Fe-Fe dumbbell states near a P substitutional atom. For each state two direction vectors are given, the orientation of the dumbbell and the direction of the displacement of the dumbbell center from the $\mathrm{P}$ atom.

\begin{tabular}{|c|c|c|}
\hline Initial state & Final state & Energy barrier $(\mathrm{eV})$ \\
\hline [110] at 1st [111] & {$[\overline{101}]$ at $2 \mathrm{nd}[001]$} & 0.608 \\
\hline$[110]$ at 1 st $[111]$ & {$[011]$ at $2 \mathrm{nd}[100]$} & 1.110 \\
\hline$[110]$ at 1 st $[111]$ & {$[110]$ at $3 r d[110]$} & 0.995 \\
\hline$[110]$ at $1 \mathrm{st}[111]$ & {$[10 \overline{1}]$ at $3 \mathrm{rd}[110]$} & 0.490 \\
\hline [110] at 1st [111] & {$[101]$ at 5 th $[111]$} & 0.432 \\
\hline$[1 \overline{1} 0]$ at $1 \mathrm{st}[111]$ & {$[101]$ at $2 \mathrm{nd}[100]$} & 0.074 \\
\hline$[1 \overline{1} 0]$ at 2 nd $[100]$ & {$[011]$ at $1 \mathrm{st}[111]$} & 0.149 \\
\hline$[110]$ at 2 nd $[100]$ & {$[10 \overline{1}]$ at 1 st $[1 \overline{1} 1]$} & 0.364 \\
\hline$[110]$ at 2 nd $[100]$ & {$[101]$ at 4 th $[311]$} & 0.288 \\
\hline$[110]$ at 2 nd $[100]$ & [011] at 4th [311] & 0.281 \\
\hline$[011]$ at $2 \mathrm{nd}[100]$ & {$[110]$ at $1 \mathrm{st}[111]$} & 0.443 \\
\hline$[011]$ at $2 \mathrm{nd}[100]$ & {$[100]$ at 4 th $[311]$} & 0.140 \\
\hline
\end{tabular}


TABLE IX. Diffusion constants and association times for the $\mathrm{P}$ interstitial defect system with a PBC cell of $11 \times 11 \times 11 \mathrm{bcc}$ unit cells. The time associated is defined here as the percentage of the time for which the interstitial defect was within $4 \AA$ of the P atom, i.e., at up to the sixth neighbor position.

\begin{tabular}{ccc}
\hline \hline Temperature $(\mathrm{K})$ & Diffusion constant $\left(\mathrm{m}^{2} \mathrm{~s}^{-1}\right)$ & Time associated \\
\hline 350 & $2.9 \times 10^{-12}$ & $100 \%$ \\
400 & $8.3 \times 10^{-12}$ & $100 \%$ \\
500 & $3.8 \times 10^{-11}$ & $99.999987 \%$ \\
600 & $1.1 \times 10^{-10}$ & $99.999627 \%$ \\
700 & $2.2 \times 10^{-10}$ & $99.993761 \%$ \\
\hline \hline
\end{tabular}

Diffusion constants calculated using the methods described in this paper can differ by orders of magnitude from those often used by nuclear engineers in rate theory models ${ }^{2}$ to assess NPV structural integrity. This suggests that the results of such calculations need to be carefully interpreted. Although the processes described here are only some of those important in determining the build-up of impurities at grain boundaries, the methodology has great promise as a means to provide a consistent set of diffusion data for such rate theory models and hence a more accurate quantification of NPV structural integrity and reactor lifetimes.

\section{ACKNOWLEDGMENTS}

The authors acknowledge funding from the EPSRC, Grants Nos. GR/R01682/01 and GR/R67699/02. One of the authors (S.M.J.G.) would like to acknowledge funding from Loughborough University for a Ph.D. studentship. The authors would like to thank Peter Flewitt, Roy Faulkner, and Zhen $\mathrm{Lu}$ for useful discussions.
${ }^{1}$ G. R. Odette and G. E. Lucas, JOM 53, 18 (2001).

${ }^{2}$ R. G. Faulkner, S. H. Song, P. E. J. Flewitt, M. Victoria, and P. Marmy, J. Nucl. Mater. 255, 189 (1998).

${ }^{3}$ Z. Lu, R. G. Faulkner, N. Sakaguchi, H. Kinoshita, H. Takahashi, and P. E. J. Flewitt, J. Nucl. Mater. 329, 1017 (2004).

${ }^{4}$ Z. Lu, R. G. Faulkner, and P. E. J. Flewitt, Acta Metall. Sin. 41, 79 (2005).

${ }^{5}$ S. H. Song, R. G. Faulkner, P. E. J. Flewitt, and L. Q. Weng, J. Mater. Sci. Technol. 21, 196 (2005).

${ }^{6}$ H. Hurchand, S. D. Kenny, C. F. Sanz-Navarro, R. Smith, and P. E. J. Flewitt, Nucl. Instrum. Methods Phys. Res. B 229, 92 (2005).

${ }^{7}$ A. V. Barashev, Philos. Mag. Lett. 82, 323 (2002).

${ }^{8}$ A. F. Voter, F. Montalenti, and T. C. Germann, Annu. Rev. Mater. Res. 32, 321 (2002).

${ }^{9}$ A. F. Voter, Phys. Rev. B 57, R13985 (1998).

${ }^{10}$ A. F. Voter, J. Chem. Phys. 106, 4665 (1997).

${ }^{11}$ M. R. Sørensen and A. F. Voter, J. Chem. Phys. 112, 9599 (2000).

${ }^{12}$ R. Marcelin, Ann. Phys. 3, 120 (1915).

${ }^{13}$ G. H. Vineyard, J. Phys. Chem. Solids 3, 121 (1957).

${ }^{14}$ A. B. Bortz, M. H. Kalos, and J. L. Lebowitz, J. Comput. Phys. 17, 10 (1975).

${ }^{15}$ G. Henkelman and H. Jónsson, J. Chem. Phys. 111, 7010 (1999).

${ }^{16}$ H. Hurchand, S. D. Kenny, C. F. Sanz-Navarro, R. Smith, and P. E. J. Flewitt, Nucl. Instrum. Methods Phys. Res. B 229, 92
(2005).

${ }^{17}$ G. Henkelman, B. P. Uberuaga, and H. Jónsson, J. Chem. Phys. 113, 9901 (2000).

${ }^{18}$ G. Henkelman and H. Jónsson, J. Chem. Phys. 115, 9657 (2001).

${ }^{19}$ R. A. Bennett, D. M. Tarr, and P. A. Mulheran, J. Phys.: Condens. Matter 15, 42 (2003).

${ }^{20}$ G. M. Crippen and H. A. Scheraga, Arch. Biochem. Biophys. 144, 462 (1971).

${ }^{21}$ R. L. Hilderbrandt, Comput. Chem. (Oxford) 1, 179 (1977).

${ }^{22}$ G. J. Ackland, M. I. Mendelev, D. J. Srolovitz, S. Han, and A. V. Barashev, J. Phys.: Condens. Matter 16, S2629 (2004).

${ }^{23}$ G. J. Ackland, D. J. Bacon, and A. F. Calder, Philos. Mag. A 75, 713 (1997).

${ }^{24}$ M. Hashimoto, Y. Ishida, R. Yamamoto, M. Doyama, and T. Fujiwara, Scr. Metall. 16, 267 (1982).

${ }^{25}$ S. M. J. Gordon, H. Hurchand, S. D. Kenny, and R. Smith, Nucl. Instrum. Methods Phys. Res. B 228, 131 (2005).

${ }^{26}$ B. P. Uberuaga, R. Smith, A. R. Cleave, F. Montalenti, G. Henkelman, R. W. Grimes, A. F. Voter, and K. E. Sickafus, Phys. Rev. Lett. 92, 115505 (2004).

${ }^{27}$ C. Domain and C. S. Becquart, Phys. Rev. B 71, 214109 (2005).

${ }^{28}$ A. V. Barashev, Philos. Mag. 85, 1539 (2005).

${ }^{29}$ C.-C. Fu, F. Willaime, and P. Ordejón, Phys. Rev. Lett. 92, 175503 (2004). 